\title{
Effect of Transcutaneous Spinal Direct Current Stimulation (TsDCS) Combined with Other Therapies on Walking Capacity in Patients with Neurological Disorders: A Systematic Review
}

\author{
Rukhsar Jabbar, Zainab Khan, Ashi Saif, and Adila Parveen* \\ Centre of Physiotherapy and Rehabilitation Sciences, Jamia Millia Islamia, New Delhi, India
}

\begin{abstract}
Introduction: Neuromodulation has been used for walking difficulty as a therapeutic approach and, as transcutaneous spinal direct current stimulation (TsDCS) is emerging as a novel tool for patients with neurological disorders when combined with transcutaneous direct current stimulation (tDCS) and/or gait training, it seems to have a promising effect; therefore, a systematic review may provide a better insight into the efficacy of the results. This systematic review aims to assess the effects of TsDCS when applied in combination with different therapies in neurological disorder patients. Methods: Databases (Pubmed, CENTRAL, and Web of Science) were used for searching studies since inception. With the guidance of reviewers, one author extracted data. Two independent reviewers assessed qualities of the randomized controlled trials (RCTs). Results: Five studies from an initial yield of 256 studies met the inclusion criteria. TsDCS might improve walking capacity when combined with tDCS and/or gait training in stroke (TsDCS with gait training and tDCS), cerebral palsy (tDCS with gait training), and cerebellar ataxia (TsDCS with tDCS). Conclusion: The result suggests that more studies are needed for concluding the therapeutic potential. Future studies should emphasize standard stimulation protocol and determining its efficacy in other outcome parameters of gait and in patients with different neurological disorders.
\end{abstract}

Keywords: transcutaneous spinal cord stimulation; noninvasive spinal direct current stimulation; gait; walking; locomotion

Citation: Jabbar, R., Khan, Z., Saif, A., \& Parveen, A. (2021). Effect of transcutaneous spinal direct current stimulation (TsDCS) combined with other therapies on walking capacity in patients with neurological disorders: A systematic review. NeuroRegulation, 8(2), 112-120. https://doi.org/10.15540/nr.8.2.112

*Address correspondence to: Dr. Adila Parveen, Assistant Professor, Centre for Physiotherapy and Rehabilitation Sciences, Jamia Millia Islamia (A central university), Jamia Nagar, New Delhi - 110025, India. Email: aparveen1@jmi.ac.in

Copyright: () 2021. Jabbar et al. This is an Open Access article distributed under the terms of the Creative Commons Attribution License (CC-BY).
Edited by:

Rex L. Cannon, PhD, SPESA Research Institute, Knoxville, Tennessee, USA

Reviewed by:

Rex L. Cannon, PhD, SPESA Research Institute, Knoxville, Tennessee, USA

Tonya Morosoli, MSc, Clínica de Neuropsicología Diagnóstica y Terapéutica, Mexico City, Mexico; ECPE, Harvard T. H. Chan School of Public Health, Boston, Massachusetts, USA

\section{Introduction}

In the last decades, noninvasive brain stimulation (NIBS) has evolved to become a valuable tool in both basic and clinical neuroscience. Various methods of NIBS such as transcutaneous direct current stimulation (tDCS), transcranial magnetic stimulation (TMS), and transcranial electrical stimulation (TES) have been widely used for diagnostic, prognostic, and even therapeutic applications in a broad range of neurological and psychiatric disorders. The rationale for using NIBS techniques lies in the possibility to modulate, in a targeted manner, the activity of different cerebral and cerebellar cortical regions, as well as the functional connections between these areas and distant brain regions, also including subcortical structures (Cosentino et al., 2018). When it comes to neurorehabilitation interventions, the improvement of the gait of the patient is an important consideration, as locomotion is the most affected factor as well as the main complication in neurological disorders which further hampers the resettlement of social and 
professional aspects of human lives (de Paz et al., 2019). The neural circuitry involved in the different aspects of gait control is very complex and includes the basal ganglia-cortical loops, the cerebellum, and structures of the brainstem and the spinal cord. This is why different approaches of NIBS have been suggested for the treatment of gait disorders in a variety of neurological disorders including Parkinson's disease, stroke, cerebellar ataxia, multiple sclerosis, cerebral palsy, and spinal cord injury (Cosentino et al., 2018). Preceding studies on the result of tDCS on gait and lower limb function have been indecisive, maybe because of the inability of tDCS to stimulate the lower limb representation in homunculus in reaching the locomotor network (Awosika et al., 2019).

The use of noninvasive modulation of spinal neurons by transcutaneous spinal direct current stimulation (TsDCS) has been variously described. In healthy people, cathodal TsDCS was recently found to improve motor unit recruitment, likely consequent to GABAergic system inhibition and direct postsynaptic neuron overexcitation, and it has been proposed as a novel therapeutic tool for managing conditions in which motor unit recruitment is reduced (Picelli et al., 2015). The segmental spinal physiology, ascending lemniscal and nociceptive pathway can be stimulated by applied direct current stimulation over the T11 spinal region so it can be viable for improving locomotor learning (Awosika et al., 2019). As well established, neuromodulation can be done at different levels of the spinal column according to the task. For example, at T11-T12 lower extremity movement is elicited as here lie the CPGs; stimulation at $\mathrm{T} 10-\mathrm{T} 11$ tends to improve the quadriceps activity, while neuromodulation at T12L1 stimulates the hamstring muscle activity (Megía García et al., 2020). At the spinal level, transcutaneous spinal stimulation increases spinal reflex activity similar to that obtained with epidural stimulation. Compared to TsDCS which is noninvasive, invasive spinal stimulation has more localized effects, but both of them activate posterior root fibers and stimulate the same region. Therefore, noninvasive nature along with other similar advantages (like that of invasive stimulation) makes the use of TsDCS more demanding and widely acceptable in clinical research (Shapkova et al., 2020). In the context of searching, methods for modulation of the spinal locomotor circuitry TsDCS can be used with other interventions for improving its efficacy, which might be limited when used alone, and can entrain the activity-dependent plasticity of spinal neuronal networks (Shapkova et al., 2020).
As locomotion is one of the major complications in patients with neurological disorders and referring to neuromodulation as an intervention, tDCS has been used for motor rehabilitation in various conditions and has prominently proven to be effective in upper extremity rehabilitation; unlike lower limb rehabilitation, this can be due to the inability of tDCS to stimulate the lower limb representation in homunculus in reaching the locomotor networks comparative to upper limb, so transcutaneous spinal stimulation has recently been introduced as a therapeutic novel approach which works on stimulating the central pattern generators (CPGs) and supraspinal networks which may prove to enhance the walking ability for patients with neurological disorders. Hence, this review will provide an insight regarding the combined effects of TsDCS with tDCS and/or gait training used for locomotion rehabilitation. Therefore, the main aim of this review is to compare the combined effects of TsDCS along with tDCS and/or gait training on locomotor ability.

\section{Methods}

\section{Search Strategy}

This review was done as per the Preferred Reporting Items for Systematic Reviews and MetaAnalyses (PRISMA) guidelines. It is registered in Prospero with the registration number CRD42021235579. We developed a search strategy to identify studies that elucidated the effects of TsDCS combined with different therapies on locomotor ability in neurological disorders. A systematic search was performed on the electronic databases MEDLINE (accessed via PubMed), CENTRAL (Cochrane Library Central Register of Controlled Trials), and Web of Science, starting from the earliest records available. Random Search items used were a combination of keywords (i.e., TsDCS, transcutaneous spinal cord stimulation, noninvasive spinal direct current stimulation, gait, walking) entered in various combinations. The keywords were combined with Boolean operators "OR" and "AND" to yield more focused results. The PRISMA flowchart is depicted in Figure 1.

\section{Eligibility Criteria}

Criteria for inclusion comprised of randomized controlled trials (RCTs) published in the English language. Subjects had to be diagnosed with the pathology of the central nervous system. Studies using TsDCS combined with other interventions and having locomotor parameters as outcome measures were included, whereas studies examining the effect of TsDCS on other outcomes such as upper limb 
motor function, pain, and spinal reflexes were excluded. Furthermore, research using other forms of invasive spinal stimulation or neuromodulation on healthy subjects and animal models were excluded. No sample size restriction was applied.

Figure 1. Prisma Flowchart Diagram.
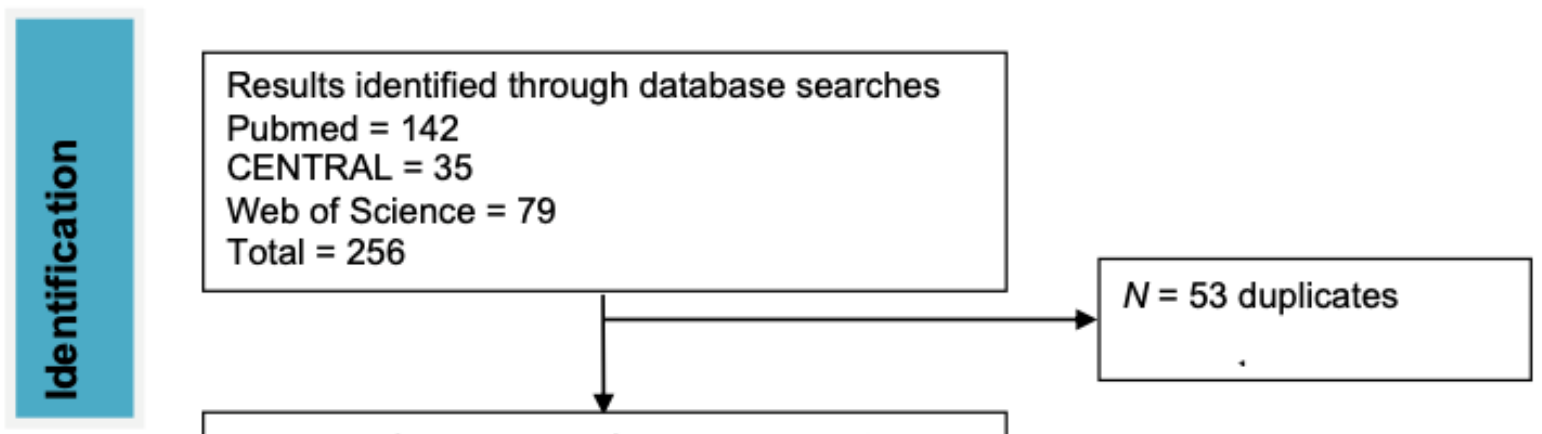

Records after removal of duplicates $n=203$
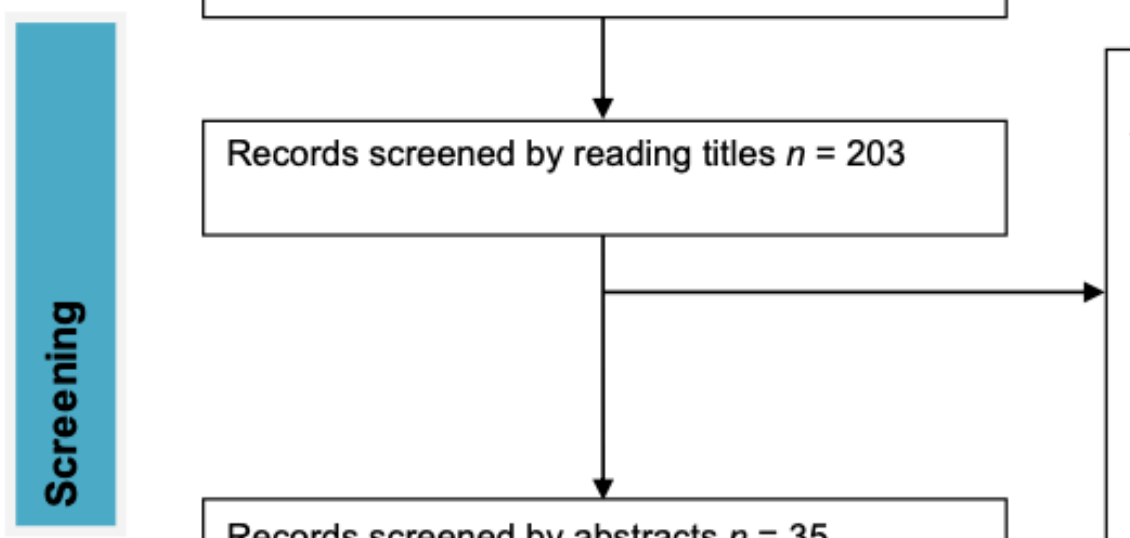

Records excluded with reason $N=168$

Records screened by abstracts $n=35$

- $\quad$ Different outcomes $=48$

- Different intervention $=67$

- Different study type $=37$

- Healthy humans $=7$

- $\quad$ Animal subject $=8$

- Different language $=1$

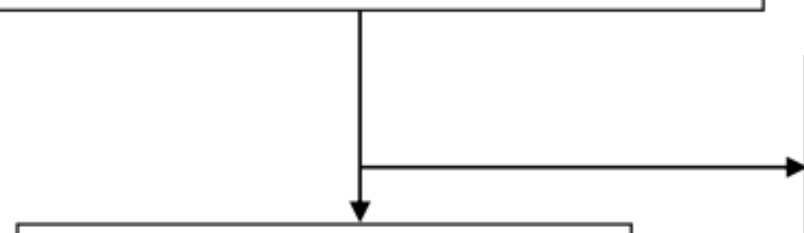

Full text articles $n=11$

$N=24$

Different outcome $n=11$

Different intervention $=21$

Ongoing trials $=3$

$N=7$ excluded

Clinical trials $=4$

Different outcome $=2$

Different intervention $=1$

Studies included $N=5$

$\mathrm{RC} T=5$ 


\section{Quality Assessment of Included Trials}

For assessing the quality of all selected RCTs, we have used an 11-point PEDro scale which evaluates the methodological quality. It assesses based on the criteria that each point system is either "Yes" or "No" answers. The scoring is such that 9-10 signifies studies of high quality, 6-8 good quality, 4-5 moderate, and below 4 poor quality. Two authors independently assessed the quality of retrieved $\mathrm{RCT}$. Any conflict was resolved by a third author.
The total score for the methodological quality is depicted in Table 1.

\section{Data Extraction}

Data on the characteristics of the trial (author, year of trial conduction, design, and duration), the participants (information on diagnosed condition), and intervention (the device used, duration, dosimetry, and safety) were extracted and depicted in Table 2, and summarized results are depicted in Table 3.

\section{Table 1}

Depicting the Quality of RCTs

\begin{tabular}{|c|c|c|c|c|c|}
\hline Article & $\begin{array}{c}\text { Benussi et al., } \\
2018\end{array}$ & $\begin{array}{l}\text { Picelli et al., } \\
\quad 2015\end{array}$ & $\begin{array}{l}\text { Picelli et al., } \\
2018\end{array}$ & $\begin{array}{l}\text { Picelli et al., } \\
2019\end{array}$ & $\begin{array}{l}\text { Solopova et } \\
\text { al., } 2017\end{array}$ \\
\hline Eligibility Criteria & Yes & Yes & Yes & Yes & Yes \\
\hline Random Allocation & Yes & Yes & Yes & Yes & Yes \\
\hline Concealed Allocation & No & Yes & Yes & Yes & Yes \\
\hline Group Similarity at Baseline & Yes & Yes & Yes & Yes & Yes \\
\hline Blinding of Subjects & Yes & Yes & No & No & No \\
\hline Blinding of Therapist & Yes & Yes & Yes & Yes & No \\
\hline Blinding of Assessor & Yes & No & Yes & Yes & No \\
\hline Dropouts $<15 \%$ & Yes & Yes & Yes & Yes & Yes \\
\hline Intention to Treat Analysis & Yes & Yes & Yes & Yes & Yes \\
\hline Between-group Differences Reported & No & Yes & Yes & Yes & No \\
\hline Point Estimate and Variability Reported & Yes & Yes & No & No & Yes \\
\hline Total Score & 8 & 9 & 8 & 8 & 6 \\
\hline Quality & Good & Excellent & Excellent & Excellent & Good \\
\hline
\end{tabular}

\section{Table 2}

Showing the Characteristics of the Selected Article

\begin{tabular}{l|lllll}
\hline Article & $\begin{array}{l}\text { Benussi et al., } \\
2018\end{array}$ & $\begin{array}{l}\text { Picelli et al., } \\
2015\end{array}$ & $\begin{array}{l}\text { Picelli et al., } \\
2018\end{array}$ & $\begin{array}{l}\text { Picelli et al., } \\
2019\end{array}$ & $\begin{array}{l}\text { Solopova et al., } \\
2017\end{array}$ \\
$\begin{array}{l}\text { Pathology; } \\
N \text { participants }\end{array}$ & $\begin{array}{l}N=20 ; \\
\text { neurodegenerative } \\
\text { ataxia }\end{array}$ & $N=30$; stroke & $\begin{array}{l}N=20 \text {; chronic } \\
\text { stroke patients }\end{array}$ & $\begin{array}{l}N=40 ; \text { supratentorial } \\
\text { stroke }\end{array}$ & $\begin{array}{l}N=28(\sim 9 \text { years); } \\
\text { with spastic cerebral } \\
\text { palsy }\end{array}$ \\
Design & $\begin{array}{l}\text { Double-blind, } \\
\text { randomized, sham- } \\
\text { controlled, } \\
\text { crossover trial }\end{array}$ & $\begin{array}{l}\text { A pilot, double-blind, } \\
\text { randomized } \\
\text { controlled trial }\end{array}$ & $\begin{array}{l}\text { A pilot, } \\
\text { single-blind, } \\
\text { randomized } \\
\text { controlled trial }\end{array}$ & $\begin{array}{l}\text { Single-center, } \\
\text { single-blind } \\
\text { RCT }\end{array}$ & Randomized \\
controlled trial
\end{tabular}


Table 2

Showing the Characteristics of the Selected Article

\begin{tabular}{|c|c|c|c|c|c|}
\hline $\begin{array}{l}\text { Area of } \\
\text { Application of } \\
\text { TsDCS and } \\
\text { TsDCS } \\
\text { Parameters } \\
\text { (Current Density; } \\
\text { Duration) }\end{array}$ & $\begin{array}{l}\text { Anode placed on } \\
\text { scalp over } \\
\text { cerebellum area ( } 2 \\
\text { cm under inion), } \\
\text { and cathode placed } \\
\text { over spinal lumbar } \\
\text { enlargement ( } 2 \mathrm{~cm} \\
\text { under T11). } \\
\text { Constant current of } \\
2 \mathrm{~mA} \text {. }\end{array}$ & $\begin{array}{l}\text { At T9 to T11, } \\
\text { Intensity }=2.5 \mathrm{~mA} \\
\text { and applied for } 20 \\
\text { min during RAGT. } \\
\text { Current ramped up } \\
\text { to } 2.5 \mathrm{~mA} \text { over a } 10- \\
\mathrm{s} \text { interval and then } \\
\text { similarly ramped } \\
\text { down at the end of } \\
\text { the stimulation. } \\
\text { Current density was } \\
0.071 \mathrm{~mA} / \mathrm{cm}^{2} \text { and } \\
\text { total charge density } \\
\text { was } 0.09 \mathrm{C} / \mathrm{cm}^{2} \text {. }\end{array}$ & $\begin{array}{l}\text { Intensity }=2.5 \mathrm{~mA} \\
\text { and applied for } 20- \\
\text { min during RAGT } \\
\text { (online stimulation), } \\
\text { combined with } \\
\text { central nervous } \\
\text { system stimulation. } \\
\text { Cathode placed over } \\
\text { spinous process of } \\
\text { the } 10 \text { th thoracic } \\
\text { vertebra (from 9th to } \\
11 \text { th) and anode } \\
\text { placed above } \\
\text { shoulder of } \\
\text { unaffected } \\
\text { hemibody. }\end{array}$ & $\begin{array}{l}\text { Intensity of TsDCS } \\
\text { set at } 2 \mathrm{~mA} \text { and } \\
\text { applied for } 20 \text { min } \\
\text { during RAGT (online } \\
\text { stimulation). For } \\
\text { patients allocated to } \\
\text { Group 1, cathode } \\
\text { positioned over } \\
\text { contralesional } \\
\text { cerebellar } \\
\text { hemisphere. }\end{array}$ & $\begin{array}{l}\text { Transcutaneous SCS } \\
\text { delivered using } 2.5- \\
\mathrm{cm} \text { round electrodes } \\
\text { placed midline at T11, } \\
\text { and } \mathrm{L} 1 \text { spinous } \\
\text { processes as } \\
\text { cathodes. } \\
\text { Biphasic rectangular } \\
1.0-\text {-ms pulses ( } 30 \\
\mathrm{~Hz} \text { ), modulated } \\
\text { frequency of } 10 \mathrm{kHz} \\
\text { were used. Intensity = } \\
10-50 \mathrm{~mA} \text { for most } \\
\text { children. }\end{array}$ \\
\hline \multirow[t]{2}{*}{ Interventions } & $\begin{array}{l}\text { Two arms: } \\
\text { Group } 1- \\
\quad \text { TsDCS + tDCS } \\
\text { Group 2- }\end{array}$ & $\begin{array}{l}\text { Three arms: } \\
\text { Group } 1- \\
\text { Anodal tDCS + } \\
\text { Sham TsDCS }\end{array}$ & $\begin{array}{l}\text { Two arms: } \\
\text { Group } 1- \\
\text { Anodal tDCS + } \\
\text { TsDCS + gait } \\
\text { training }\end{array}$ & $\begin{array}{l}\text { Two arms: } \\
\text { Group 1- } \\
\text { Contralesional } \\
\text { tDCS + TsDCS }\end{array}$ & $\begin{array}{l}\text { Two arms: } \\
\text { Group } 1- \\
\text { TsDCS + } \\
\text { Locomotor training }\end{array}$ \\
\hline & Sham stimulation & $\begin{array}{l}\text { Group } 2- \\
\text { Sham tDCS + } \\
\text { Cathodal TsDCS } \\
\text { Group } 3- \\
\quad \text { Anodal tDCS + } \\
\text { Cathodal TsDCS }\end{array}$ & $\begin{array}{l}\text { Group } 2- \\
\text { Cathodal tDCS + } \\
\text { TsDCS + gait } \\
\text { training }\end{array}$ & $\begin{array}{l}\text { Group } 2 \text { - } \\
\text { Ipsilesional tDCS + } \\
\text { TsDCS }\end{array}$ & $\begin{array}{l}\text { Group } 2- \\
\quad \text { Locomotor training }\end{array}$ \\
\hline Variables & $\begin{array}{l}\text { SARA, ICARS, 9- } \\
\text { Hole Peg Test, 8- } \\
\text { Min Walking Time }\end{array}$ & $\begin{array}{l}\text { 6MWT, FAC, MI, } \\
\text { AS, SGP }\end{array}$ & $\begin{array}{l}6 \mathrm{MWT} \text {, selected as } \\
\text { a measure of } \\
\text { walking capacity; } \\
\text { FAC, } \\
\text { MI, AS; SGP, } \\
\text { evaluated with the } \\
\text { GAITRite system }\end{array}$ & $\begin{array}{l}\text { 6MWT, FAC, MI, AS, } \\
\text { SGP }\end{array}$ & $\begin{array}{l}\text { Modified AS; L-ROM } \\
\text { test, as directed by } \\
\text { Lokomat software; } \\
\text { L-FORCE test of } \\
\text { Lokomat software }\end{array}$ \\
\hline
\end{tabular}

Note. RAGT = robot-assisted gait training; 6MWT = 6-Min Walk Test; FAC = Functional Ambulation Category; MI = Motricity Index leg subscore; AS = Asworth scale; SGP = Spatiotemporal gait parameter; SARA = Scale for Assessment and Rating of Ataxia, ICARS = International Cooperative Ataxia Rating Scale.

\section{Results}

We developed a search strategy and included three databases. 256 studies were yielded, out of which 142 were in PubMed, 35 in CENTRAL, and 79 in the Web of Science. After removing 53 duplicates, 203 articles remained. On screening of titles and abstracts, 11 full-text articles were retrieved from the remaining and, finally, 5 were selected as per inclusion criteria. Table 3 shows the most relevant characteristics of selected articles. 
Table 3

Showing the Results of Selected RCTs

Article Results

Benussi et al., 2018

Cerebello-spinal tDCS decreases signs in ataxia patients and restores motor cortex inhibition by cerebellar structures.

Picelli et al., 2015

Anodal tDCS when used with cathodal TsDCS might be beneficial for enhancing the effects of robotic gait training in chronic stroke.

Picelli et al., 2018

Cathodal tDCS over contralateral cerebellar hemispheres when used along with cathodal TsDCS may be beneficial to enhance the effects of robot-assisted gait training in chronic stroke.

Picelli et al., 2019

Cathodal tDCS over contralateral or ipsilateral cerebellar hemisphere when used along with cathodal TsDCS might improve gait with similar effects in both combinations in stroke patients.

Solopova et al., 2017

The gait ability can be significantly enhanced with TsDCS when combined with locomotor training comparative to locomotive training alone.

\section{Participants}

The sample that is part of this review was comprised of a total of 141 patients with different types of pathologies: 93 subjects with chronic stroke in three studies (Picelli et al., 2015, 2018, 2019), 28 subjects with cerebral palsy (Solopova et al., 2017), and 20 subjects with neurodegenerative ataxia (Benussi et al., 2018).

\section{Stimulation Patterns and Parameters of TsDCS} In terms of electrode size for TsDCS, it was 23.75 $\mathrm{cm}^{2}$ (Picelli et al., 2018, 2019), $35 \mathrm{~cm}^{2}$ (Picelli et al., 2015), $8 \times 6 \mathrm{~cm}^{2}$ (Benussi et al., 2018), and $5 \times 8$ $\mathrm{cm}^{2}$ (Solopova et al., 2017), respectively. In three of the studies, the cathode was placed over the spinous processes of the thoracic vertebra (from 9th to 11th) and the anode was placed above the shoulder of the unaffected hemibody (Picelli et al., 2015, 2018, 2019); in the other two studies, the cathode was placed midline at T11 and at L1 spinous processes (Solopova et al., 2017) and was placed over the spinal lumbar enlargement $(2 \mathrm{~cm}$ under T11; Benussi et al., 2018).

The current intensities for cathodal TsDCS were 2.5 $\mathrm{mA}$ (Picelli et al., 2015, 2018, 2019), 10 to $50 \mathrm{~mA}$ (Solopova et al., 2017), and $2.0 \mathrm{~mA}$ (Benussi et al., 2018), respectively, and current density was 0.071 $\mathrm{mA} / \mathrm{cm}$ (Picelli et al., 2015, 2018, 2019), whereas the total charge density was $0.09 \mathrm{C} / \mathrm{cm}^{2}$ (Picelli et al., 2015, 2018, 2019) and $0.042 \mathrm{~mA} / \mathrm{cm}^{2}$ (Benussi et al., 2018) for respective studies. As far as the duration of TsDCS was concerned, the sessions were 20 min for all studies (Benussi et al., 2018; Picelli et al., 2015, 2018, 2019; Solopova et al., 2017).
All of the included studies used TsDCS along with other therapies, such as TsDCS with tDCS and gait training (Picelli et al., 2015, 2018, 2019), cerebellar tDCS with TsDCS (Benussi et al., 2018), and TsDCS with locomotor training only (Solopova et al., 2017).

\section{Combined Therapies Protocol}

Transcranial Direct Current Stimulation (tDCS)

Parameters. Among the five studies, four combined tDCS with TsDCS (Benussi et al., 2018; Picelli et al., $2015,2018,2019)$. In one study, the electrode size was $7 \times 5 \mathrm{~cm}, 35 \mathrm{~cm}^{2}$, and the anode was placed on a motor area as per EEG system on ipsilesional area and cathode over another side. For anodal tDCS stimulation, the current intensity was $2 \mathrm{~mA}$ for 20 min (along with gait training) and charge density was $0.07 \mathrm{C} / \mathrm{cm}^{2}$ (Picelli et al., 2015); while in another study, the electrode size was about $4 \mathrm{~cm}$ in diameter, $12.56 \mathrm{~cm}^{2}$ in area, and the anode was placed on the ipsilateral side over the motor cortex (M1), as per EEG only, and the cathode was positioned over the orbit of the other side (Picelli et al., 2018). In another included trial (Picelli et al., 2019), the electrode size was similar to the previously mentioned study (Picelli et al., 2018), but the cathodal electrode was placed over the cerebellar hemisphere while the anode was over the buccinator muscle on the same side (Picelli et al., 2019). The current intensity, which was $2 \mathrm{~mA}$, and the duration of $20 \mathrm{~min}$ were the same for all three studies (Picelli et al., 2015, 2018, 2019). In the fourth study, the anode was placed over the cerebellar region (electrode size $7 \times 5 \mathrm{~cm}^{2}$, current density $0.057 \mathrm{~mA} / \mathrm{cm}^{2}$ ) and used tDCS as an additional therapy with TsDCS (Benussi et al., 2018). 
Gait Training Protocol. The gait training was combined in four studies with TsDCS intervention (Picelli et al., 2015, 2018, 2019; Solopova et al., 2017), and in three studies among them, the criteria were similar. The robot-assisted gait training was divided into two parts: first $10 \%$ weight was supported for $10 \mathrm{~min}$ and speed was $1.5 \mathrm{~km} / \mathrm{hr}$, then speed was increased to $2 \mathrm{~km} / \mathrm{hr}$ and no body weight was supported for the next $10 \mathrm{~min}$ (Picelli et al., 2015, 2018, 2019). In one study (Solopova et al., 2017), the duration of locomotor training was $40 \mathrm{~min}$ at a treadmill speed of around $1 \mathrm{~km} / \mathrm{hr}$ and the stimulation was switched off after $20 \mathrm{~min}$.

\section{Sham Group/Other Comparison Group Protocols} In all five selected studies, the experimental group was either compared to the control group (Solopova et al., 2017) or with other intervention groups (sham stimulation or stimulation over the contralateral side; Benussi et al., 2018; Picelli et al., 2015, 2018, 2019). The protocol parameters for sham tDCS in one of the studies was that it was applied for 2 min in starting and $30 \mathrm{~s}$ in ramp down fashion at the end at the same intensity as anodal stimulation (Picelli et al., 2015); while in the other two studies, the tDCS placement was over the contralesional side for comparison (Picelli et al., 2018, 2019).

One included study (Benussi et al., 2018) reported the electrode positioning was similar to the experimental group, but the current was ramped down after $5 \mathrm{~s}$. Additionally, a reference group was also included in which TMS was applied on healthy controls. The parameters for TMS were that the coil was placed over the motor cortex area and the handle was directed at 45 degrees laterally and posteriorly to the longitudinal plane (Benussi et al., 2018). While only in a single study, the control group received only locomotor training with Lokomat (Hocoma AG, Switzerland) for $40 \mathrm{~min}$ without TsDCS (Solopova et al., 2017) and was compared to the experimental group that received both gait training and TsDCS.

\section{Recorded Variables and Their Effect}

Patients were evaluated before treatment (T0), immediately after treatment (primary endpoint; T1), at 2 weeks (T2), and at 4 weeks (T3) of follow-up in three studies (Picelli et al., 2015, 2018, 2019). In another included trial, the assessment was taken as in the first phase, at baseline, at 2 weeks after stimulation, at 1 month, and 3 months (Benussi et al., 2018), while the fifth study evaluated before and after comparisons with no follow-up (Solopova et al., 2017).
Walking Capacity. The most used scales were the 6-Min Walk Test (6MWT; Picelli et al., 2015, 2018, 2019), SARA (Scale for Assessment and Rating of Ataxia), and ICARS (International Cooperative Ataxia Rating Scale; Benussi et al., 2018), and GMFM-88 (Solopova et al., 2017) for evaluating the walking capacity in different neurological conditions. Two studies showed a statistically significant difference between groups at T0, T1, and T2 followups but were not maintained at T3 follow-up (Picelli et al., 2015, 2019). In one included trial (Solopova et al., 2017), the change in total GMFM-88 score was significantly correlated with the increase of walking (dimension E in GMFM-88). Similarly, another study (Benussi et al., 2018) reported improvement in SARA and ICARS scores in terms of gait between groups, but one included trail (Picelli et al., 2018) scoring did not differ between the two groups.

Spasticity. The modified Ashworth scale was used mainly in all included studies for spasticity assessment (Picelli et al., 2015, 2018, 2019; Solopova et al., 2017). In four studies, there was no change in spasticity between groups (Picelli et al., 2015, 2018, 2019; Solopova et al., 2017).

Lower Limb Strength. The lower limb strength was assessed by the Motricity Index (MI) leg subscore in all three studies (Picelli et al., 2015, 2018, 2019). Though in two studies there was no improvement found between the groups at T1-T0, T2-T0, T3 follow-up (Picelli et al., 2015, 2019), in a single study, there was a significantly greater improvement in affected lower limb motricity (Picelli et al., 2018).

The Cadence of Stride and Single/Double Support Ratio. Three studies assessed cadence and single/double ratio by GAITRite system (CIR Systems, Inc., Franklin, NJ; Picelli et al., 2015, 2018, 2019). Though in two studies there was no improvement in cadence and single/double support ratio (Picelli et al., 2018, 2019), in one included study (Picelli et al., 2015), significant differences in cadence were found between the groups at the T1T0 and T2-T0 follow-ups but not at the T3-T0 evaluation. Also, no significant difference in the ratio between single and double support duration was found between the groups at the T1-T0, T2-T0, and T3-T0 follow-up.

\section{Discussion}

This systematic review aimed to determine the effectiveness of TsDCS combined with tDCS and/or gait training on locomotor rehabilitation in neurological disorders. To the best of our 
knowledge, this is the first systematic review based on data from 141 participants evaluating the effects of TsDCS with tDCS and/or gait training on gait rehabilitation in patients with neurological disorders. Out of the five RCTs, four studies (Benussi et al., 2018; Picelli et al., 2015, 2019; Solopova et al., 2017) showed that TsDCS along with tDCS and/or gait training improves walking capacity in patients with neurological disorders. However, one study (Picelli et al., 2018) did not show any improvement in walking ability, which is the primary outcome, and regarding the secondary outcomes, there are inconclusive results as not all studies have shown significant changes. Specifically, no improvement in spasticity in was any of the studies (Benussi et al., 2018; Picelli et al., 2015, 2018, 2019; Solopova et al., 2017), while cadence (Picelli et al., 2015) and single and double support ratio (Picelli et al., 2018) showed an improvement in respective studies. Overall, our review suggests that TsDCS helps in improving the efficacy of tDCS with/or gait training for promoting walking ability.

In all included studies, the current intensity of TsDCS ranges from 2.0 to $2.5 \mathrm{~mA}$, except in one study by Solopova et al. (2017), in which it is $10 \mathrm{~mA}$ to $50 \mathrm{~mA}$; the treatment session was $20 \mathrm{~min}$ in all studies. However, the current density is used in varied patterns. The site for application is from T9 to T11-L1 in all included studies (Benussi et al., 2018; Picelli et al., 2015, 2018, 2019; Solopova et al., 2017) and none of the assessed studies reported adverse effects. TsDCS with anodal tDCS has shown to improve the gait in post-stroke patients, as improvements in walking function have been associated with changes in the activation of cortical areas involved in motor control and strengthening of descending input from the brain (Picelli et al., 2015); while in another study also on stroke population (Picelli et al., 2019), TsDCS was combined with cathodal tDCS and stimulation effects were compared between lesional and contralesional sides. Both have shown to improve walking capacity, and no superiority was found in between ipsilesional and contralesional application of cerebellar tDCS combined with TsDCS, as both equally improve walking ability. This can be due to the peculiar bilateral representation of gait function (walking is associated with activation of the primary motor cortex bilaterally, the supplementary motor area, and somatosensory cortex compared with contralateral M1 focus during isolated arm movements), which is considered (Picelli et al., 2018). Another included study on cerebral palsy patients (Solopova et al., 2017) reported a stable improvement of motor functions in children and, in this study, the TsDCS is combined with gait training and proposes that some functional improvement of the spinal supraspinal networks that control locomotor functions were induced.

No change in spasticity was observed in any of the studies (Benussi et al., 2018; Picelli et al., 2015, 2018, 2019; Solopova et al., 2017). As far as TsDCS mechanism of action is concerned regarding spasticity, one of the included studies by Picelli et al. (2015) reported that TsDCS makes motor neurons more susceptible to activation but less responsive to enhance activity that decreases the interneuronal network. Another outcome measure lower limb motricity (which is used for assessment of lower limb strength) has been improved in one included study (Picelli et al., 2018); improvement in motricity might be because of the cerebellar stimulation combined with TsDCS, which has main effects in bringing the change related to inhibition of thalamocortical pathway which is involved for producing new gait patterns (Picelli et al., 2018).

Although TsDCS mechanism of action is a topic of discussion, a growing body of evidence advocates that TsDCS interferes with cortical, corticospinal, and spinal motor output in humans (Ardolino et al., 2021). TsDCS has been shown to influence the ascending and descending spinal pathways and spinal reflex excitability, with increasing evidence that it can induce prolonged functional neuroplastic changes (Benussi et al., 2018).

It appears that the TsDCS when used with other interventions (tDCS and/or gait training) can elicit adaptive neuroplasticity on spinal stimulation (Benussi et al., 2018). This idea is also in line with the review of Megía García et al. (2020), which indicated that TsDCS is a practicable option for increasing voluntary motor response of the upper and lower limbs, trunk stability, and improvement of function and quality of life of patients with spinal cord injury, even though they have included case series and case report having a low level of evidence. Also, another systematic review of Grecco et al. (2015), considered transcutaneous spinal stimulation as a promising therapeutic tool for patients suffering from spinal cord injury. Our review will add to the current knowledge that, apart from spinal cord injury patients, TsDCS when combined with tDCS and/or gait training can be used to increase the efficacy of therapies used for gait rehabilitation in various neurological disorders. 


\section{Limitations of Review}

The main limitation of this systematic review is the limited number of trials conducted for a great diversity of outcomes of gait and the pattern of application of stimulation. Also, concluding is a complicated insight as we have studied various neurological pathologies. The numbers of subjects studied are less; nevertheless, larger RCTs must be conducted to demonstrate its full therapeutic potential. All studies have combined intervention with TsDCS, so effects of TsDCS specifically cannot be stated. Promoting neuroplasticity as an outcome for stimulation has been a recent approach and, if results of this approach are confirmed in larger trials, then this crucial therapy might be beneficial as a therapeutic approach for several neurological conditions.

\section{Conclusion}

Overall, our review suggests that TsDCS can be used in improving walking ability when combined with tDCS and/or gait training for locomotor rehabilitation, although in few included studies, the effects were not observed in succeeding follow-ups (Picelli et al., 2018, 2019). This discovery can be considered in analyzing the duration of application of TsDCS as an intervention. Also, all the included studies involved patients with different neurological disorders, so accurate magnitude of the effect and result cannot be concluded for one particular disorder; hence, more studies are needed for having a clear picture of the effects of TsDCS on different neurological disorders.

\section{Author Declarations}

There was no grant support for this review. There is no financial interest in a company or its competitor of a product discussed in the review. Authors declare no conflict of interest.

\section{References}

Ardolino, G., Bocci, T., Nigro, M., Vergari, M., Di Fonzo, A., Bonato, S., Cogiamanian, F., Cortese, F., Cova, I., Barbieri, S., \& Priori, A. (2021). Spinal direct current stimulation (tsDCS) in hereditary spastic paraplegias (HSP): A shamcontrolled crossover study. The Journal of Spinal Cord Medicine, 44(1), 46-53. https://doi.org/10.1080 /10790268.2018.1543926

Awosika, O. O., Sandrini, M., Volochayev, R., Thompson, R. M., Fishman, N., Wu, T., Floeter, M. K., Hallett, M., \& Cohen, L. G. (2019). Transcutaneous spinal direct current stimulation improves locomotor learning in healthy humans. Brain Stimulation, 12(3), 628-634. https://doi.org/10.1016 /j.brs.2019.01.017.

Benussi, A., Dell'Era, V., Cantoni, V., Bonetta, E., Grasso, R., Manenti, R., Cotelli, M., Padovani, A., \& Borroni, B. (2018).
Cerebello-spinal tDCS in ataxia A randomized, double-blind, sham-controlled, crossover trial. Neurology, 91(12), E1090E1101. https://doi.org/10.1212/WNL.0000000000006210

Cosentino, G., Brighina, F., Alfonsi, E., \& Sandrini, G. (2018). Effects of neuromodulation on gait. In G. Sandrini, V. Homberg, L. Saltuari, N. Smania, \& A. Pedrocchi (Eds.), Advanced Technologies for the Rehabilitation of Gait and Balance Disorders. Biosystems and Biorobotics (Vol. 19, pp. 367-397). Switzerland: Springer International Publishing. https://doi.org/10.1007/978-3-319-72736-3_26

de Paz, R. H., Serrano-Muñoz, D., Pérez-Nombela, S., BravoEsteban, E., Avendaño-Coy, J., \& Gómez-Soriano, J. (2019). Combining transcranial direct-current stimulation with gait training in patients with neurological disorders: A systematic review. Journal of NeuroEngineering and Rehabilitation, 16(1), 114. https://doi.org/10.1186/s12984-019-0591-z

Grecco, L., Li, S., Michel, S., Castillo-Saavedra, L., Mourdoukoutas, A., Bikson, M., \& Fregni, F. (2015). Transcutaneous spinal stimulation as a therapeutic strategy for spinal cord injury: State of the art. Journal of Neurorestoratology, 3, 73-82. https://doi.org/10.2147 /JN.S77813

Megía García, A., Serrano-Muñoz, D., Taylor, J., Avendaño-Coy, J., \& Gómez-Soriano, J. (2020). Transcutaneous spinal cord stimulation and motor rehabilitation in spinal cord injury: A systematic review. Neurorehabilitation and Neural Repair, 34(1), 3-12. https://doi.org/10.1177/1545968319893298

Picelli, A., Brugnera, A., Filippetti, M., Mattiuz, N., Chemello, E., Modenese, A., Gandolfi, M., Waldner, A., Saltuari, L., \& Smania, N. (2019). Effects of two different protocols of cerebellar transcranial direct current stimulation combined with transcutaneous spinal direct current stimulation on robotassisted gait training in patients with chronic supratentorial stroke: A single blind, randomized controlled trial. Restorative Neurology and Neuroscience, 37(2), 97-107. https://doi.org /10.3233/RNN-180895

Picelli, A., Chemello, E., Castellazzi, P., Filippetti, M., Brugnera, A., Gandolfi, M., Waldner, A., Saltuari, L., \& Smania, N. (2018). Combined effects of cerebellar transcranial direct current stimulation and transcutaneous spinal direct current stimulation on robot-assisted gait training in patients with chronic brain stroke: A pilot, single blind, randomized controlled trial. Restorative Neurology and Neuroscience, 36(2), 161-171. https://doi.org/10.3233/RNN-170784

Picelli, A., Chemello, E., Castellazzi, P., Roncari, L., Waldner, A., Saltuari, L., \& Smania, N. (2015). Combined effects of transcranial direct current stimulation (tDCS) and transcutaneous spinal direct current stimulation (tsDCS) on robot-assisted gait training in patients with chronic stroke: A pilot, double blind, randomized controlled trial. Restorative Neurology and Neuroscience, 33(3), 357-368. https://doi.org /10.3233/RNN-140474

Shapkova, E. Y., Pismennaya, E. V., Emelyannikov, D. V., \& Ivanenko, Y. (2020). Exoskeleton walk training in paralyzed individuals benefits from transcutaneous lumbar cord tonic electrical stimulation. Frontiers in Neuroscience, 14, 416. https://doi.org/10.3389/fnins.2020.00416

Solopova, I. A., Sukhotina, I. A., Zhvansky, D. S., Ikoeva, G. A., Vissarionov, S. V., Baindurashvili, A. G., Edgerton, V. R., Gerasimenko, Y. P., \& Moshonkina, T. R. (2017). Effects of spinal cord stimulation on motor functions in children with cerebral palsy. Neuroscience Letters, 639, 192-198. https://doi.org/10.1016/j.neulet.2017.01.003

Received: April 12, 2021

Accepted: June 1, 2021

Published: June 30, 2021 